Fluorescence in situ hybridization study that revealed a deletion on chromosome 22q11.2 zone. Therapy with calcium was undertaken at first by intravenous infusion and orally afterwards; high levels of calcium were needed to normalize serum calcium.

Molecular evaluation of the parents showed no deletion in the $22 q 11.2$ zone, allowing for the diagnosis of a de novo deletion in the index case.

The importance of this report relies on the fact that the patient, despite clinical suspicions of VCFS, remained asymptomatic until late childhood, presenting with no renal dysfunction, immunological abnormalities or cardiac malformations.

Considering this hypothesis and making an early diagnosis is important both for implementing timely clinical evaluation and dietary supplementation if needed and for family planning.

\section{DIFFERENT FACES OF CHRONIC AUTOIMMUNE THYROIDITIS IN CHILDHOOD AND ADOLESCENCE}

doi:10.1136/archdischild-2012-302724.0664

L Saranac. Pediatric Clinic, University Clinical Centre Nis, Nis, Serbia

Chronic autoimmune thyroiditis (CAT) is multifaced disease. Its incidence has increased dramatically over the past few decades afflicting up to $2 \%$ of the general population. CAT as autoimmune disorder results from complex interplay of genetic, environmental and endogenous factors. In nonendemic goitre areas, CAT is the most common cause of acquired hyothyroidism.

Unlike overt goitrogenic form of disease, atrophic one remains hidden or misdiagnosed for years.

Some children expirience all thyroid dysfunction types during natural course of the disease; mild hyperthyroidism at diagnosis (hashitoxicosis), euthyroid state, and gradual progression from subclinical to overt hypothyroidism.

The clinical manifestations of acquired hypothyroidism (AH) in childhood differ from those in adults. The classic manifestation also occur in children, but are not so prominent. Instead, the most important sign of $\mathrm{AH}$ in childhood is growth failure. Weight tends to increase and in most instances weight for age is greater than height for age. The retardation of bone age in AH usually equals or exceeds the retardation in linear growth. Pubertal disorders: delayed, or even precocious puberty are always present.

Another intriguing form of dysfunction in CAT could be the subclinical hypothyroidism with mixed signs of hypo and hyperfunction (autoimmune dysthyroidism). Thus, clinical features do not always correspond to hormonal status. The reasons for diagnostic pitfalls, because of clinical ambiguity are challenging for pediatricians and endocrinologists.

\section{RETROPHARYNGEAL ABSCESS AS A PITFALL IN THE DIAGNOSIS OF KAWASAKI DISEASE: CASE REPORT AND REVIEW OF THE LITERATURE}

doi:10.1136/archdischild-2012-302724.0665

${ }^{1} \mathrm{~L}$ Da Dalt, ${ }^{1} \mathrm{P}$ Berlese, ${ }^{1} \mathrm{ME}$ Cavicchiolo, 'MS Strafella, ${ }^{2} \mathrm{E}$ Trincia, ${ }^{2}$ I Inches, ${ }^{3} \mathrm{~S}$ Bressan. 'Pediatric Unit; ${ }^{2}$ Neuroradiology Unit, Treviso Hospital, Treviso; ${ }^{3}$ Department of Pediatrics, University of Padova, Padova, Italy

Kawasaki disease $(\mathrm{KD})$ is an acute self-limiting vasculitis of childhood of unknown etiology. We report the case of a patient with $\mathrm{KD}$ whose initial presentation mimicked a retropharyngeal abscess (RA) and review the literature on this topic. Our child, a 4 year old girl, presented with fever ( $<24$ hours) and clinical, laboratory and MRI findings.

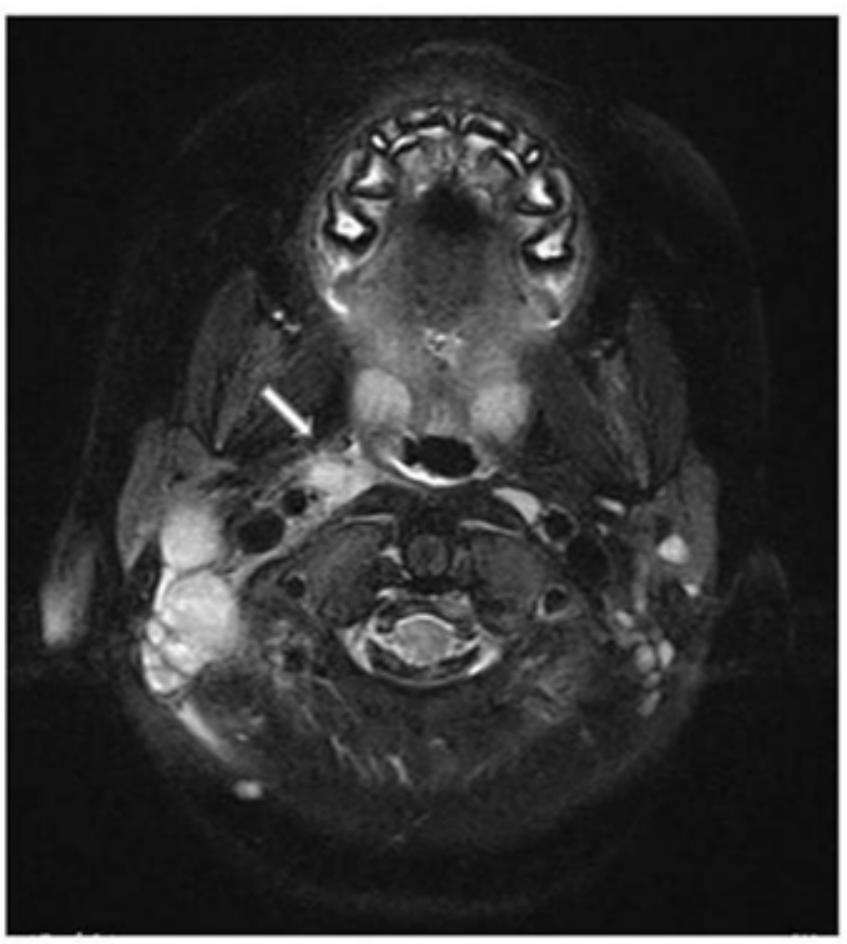

Abstract 665 Figure 1

Consistent with RA. She received prompt intravenous antibiotic therapy with no clinical improvement. Only repeated accurate physical examinations, with early appreciation of subsequent clinical findings consistent with $\mathrm{KD}$, allowed for early diagnosis and proper treatment with intravenous immunoglobulin. In literature sixteen cases of $\mathrm{KD}$ mimicking RA have been reported. Fever and deep neck infection like symptoms were the only clinical findings at admission in 14 (87.5\%) children. All children had a neck CT scan performed showing findings suggestive of RA. All children were promptly started intravenous antibiotic therapy without clinical improvement and five patients (31\%) underwent unproductive surgical drainage of the retropharyngeal space. Twelve patients $(80 \%)$ received a diagnosis of $\mathrm{KD}$ after 7 or more days since onset of fever and 4 after 10 or more days. Coronary aneurisms were found in 2 patients, both of whom had a delayed KD diagnosis after 10 or more days since fever onset.

Conclusion Pediatricians should be aware of atypical KD presentations mimicking RA. Early diagnosis of $\mathrm{KD}$ is pivotal for preventing cardiac complications, as well as avoiding the risk associated to unnecessary surgical intervention.

\section{PULMONARY ABNORMALITIES IN CHILDREN AND ADOLESCENTS WITH TYPE 1 DIABETES MELLITUS}

doi:10.1136/archdischild-2012-302724.0666

${ }^{1} \mathrm{GC}$ Yılmaz, 'P Boran, '2L Yılmaz, 'E Tutar, 'G Tokuc. 'Dr. Lutfi Kirdar Kartal Research and Training Hospital, 2nd Clinic of Pediatrics; ${ }^{2}$ Dr. Lutfi Kirdar Kartal Research and Training Hospital, Department of Family Medicine, Istanbul, Turkey

Background and Aims Few studies are available on pulmonary function abnormalities in children with diabetes with controversial results. Spirometric abnormalities and reduction of lung diffusing capacity for carbon monoxide (DLCO) have been reported.

A cross sectional study was designed to assess whether children and adolescents with type 1 diabetes have pulmonary dysfunction. 
Methods Spirometry measurements were performed and DLCO was measured. The final data analysis was conducted on 57 diabetics (mean age $14.4+3.09$ years, 31 males) and 40 healthy controls (mean age $13.6+2.2$ years, 19 males).

Results Although FVC, FEV1, FEV1/FVC of diabetics were lower than in control, significant statistical analysis was found only for FEF 25-75. Statistically significant differences between diabetic and control girls was noted with lower FEV1, lower FEV1/FVC, lower FEF25-75 and reduced DLCO/VA values in diabetic girls. Almost no correlation was found for diabetes duration, HbA1c, HRV indices and pulmonary function variables.

Conclusions In conclusion, the results of our study indicate subclinical lung impairment in children with T1DM, with significantly reduced FEF 25-75 compared to control subjects, indicating early small airway obstructive pattern. In this study, statistically significant differences between diabetic and control girls was noted, suggesting obstructive airway disease, according to the spirometric evaluation, but restrictive derangement indicated by reduced DLCO/VA. Since total lung capacity was not measured in our study, we can not talk about a restrictive pattern according to reduced DLCO/VA, but gender appeared a significant determinant for pulmonary dysfunction.

\section{GASTROESOPHAGEAL PH CHARACTERIZES FUNCTIONAL AIRWAYS RESPONSE IN ASTHMATIC CHILDREN}

doi:10.1136/archdischild-2012-302724.0667

C Pacchiarotti, M Barreto, A Bozzone, M Campisano, S Fedeli, A Macari, F La Penna, F Guglielmi, MP Villa. Sapienza University of Roma, Roma, Italy

Background Gastroesophageal acidity $(\mathrm{GE} \mathrm{pH})$ could be related to lung function and airway response to exercise in children with lower airways disease.

Aims To compare GE pH with lung function before and after exercise in asthmatic and non-asthmatic outpatients with recurrent respiratory symptoms.

Methods 16 asthmatic and 15 non-asthmatic patients (aged 4 5-15.7 yr; M/F 23/8) did lung function before and after 24-h GE pH monitoring $\left(\mathrm{GE} \mathrm{pH}_{24}\right)$. Subjects also undergone exercise-testing (treadmill) 1 hour before GE catheter removal. GE pH was also analysed for 6-minute intervals each (before, during and after exercise).

Results GE $\mathrm{pH}{ }_{24}$ levels did not correlate with exercise outcomes, nor yielded statistical differences between patient groups. Instead, GE $\mathrm{pH}_{24}$ correlated positively with baseline $\mathrm{FEV}_{1} \%$ in asthmatic subjects $(r=0.66, p=0.006)$ whereas correlated negatively in nonasthmatic subjects $(\mathrm{r}=-0.61, \mathrm{p}=0.016)$. Median (interquartile range, IOR) values of GE $\mathrm{pH}$ fall during exercise in asthmatic but not in non-asthmatic children (-4.64, IOR:10.2 vs 0.00, IOR:5.64, p=0.033). Conversely, GE $\mathrm{pH}$ values increased 1 hour after exercise only in asthmatic children (5.80, IOR:7.73 vs 0.00, IQR:9.82, $\mathrm{p}=0.012$ ).

Conclusion Our data suggest a characteristic relationship between $\mathrm{GE} \mathrm{pH}$, lung function and airway response to exercise in asthmatic children; whether GE $\mathrm{pH}$ is cause or consequence of these bronchial changes remains to be established.

\section{YOUNGER CHILDREN ARE MORE RELIABLE IN ESTIMATING THE SEVERITY OF ASTHMA ATTACK COMPARING TO PARENTS AND OLDER CHILDREN}

doi:10.1136/archdischild-2012-302724.0668

${ }^{1} \mathrm{D}$ Stamatovic, ${ }^{2} \mathrm{~K}$ Starinac, ${ }^{3} \mathrm{Z}$ Zivkovic, ${ }^{1} \mathrm{~A}$ Stamatovic. ${ }^{1}$ Private Pediatric Practice 'Primum Vivere'; 'Dispensary for Children Health Care, Krusevac; ${ }^{3}$ Faculty of Medicine, Pristina University, Kosovska Mitrovica, Serbia
Background and Aims Many studies emphasize that child's perception of asthma symptoms is not reliable.

We assessed correlation between objective and subjective asthma attack estimation and analyzed factors that affect objectivity.

Methods Independently from parents, 33 children ages 5-18 years estimated asthma attack severity from $0-10$ by visual analog scale score (VASS) - subjective estimation. Objective estimation for each child was done in two ways: comparing absolute values of lung function parameters (LFP) with Zapletal's norms (ZN): LFP\%ZN by Jeager Flowscreen spirometer, and with individual best values during optimal disease control, individual norms (IN): LFP\%IN. We analyzed the correlation between subjective and objective estimations.

Results VASS of children and parents were not in mutual correlation. VASS of children and parents were not in correlation with spirometric estimation (LFP\%ZN). In younger children, correlation becomes statistically significant if we use IN instead of $Z N$ (LFP\%IN): $\mathrm{r}=-0.45, \mathrm{p}=0.049$ (FEV1); $\mathrm{r}=-0.52, \mathrm{p}=0.020$ (MEF50); $r=-0.6, p=0.005$ (MEF25). VASS of their parents were not in correlation with LFP\%IN. In 15 of 20 cases, IN of younger children were higher than $Z \mathrm{~N}$, thus more valid. On the contrary, IN of older children were mainly below ZN (in 10 of 13), thus less reliable for estimation. In this group there was no correlation regardless of applied norm and whether the estimation was done by child or a parent.

Conclusions Children ages 5-10 years are more reliable in estimating the severity of asthma attack than older children and their parents who are adapted to lower child's possibilities.

\section{MONITORING THE ASTHMATIC CHILDREN, A PROSPECTIVE STUDY}

doi:10.1136/archdischild-2012-302724.0669

${ }^{1} \mathrm{MBI}$ Octavia, ${ }^{2} \mathrm{~N}$ Bogdan, ${ }^{3} \mathrm{NM}$ Leonida, 'S Iulia, ${ }^{4} \mathrm{M}$ Rodica, ${ }^{5} \mathrm{H}$ Mirela, ${ }^{6} \mathrm{~S}$ Claudia, ${ }^{7} \mathrm{M}$ lonela, ${ }^{8} \mathrm{~K}$ Otto, ${ }^{5} \mathrm{M}$ Melaniea, ${ }^{2} \mathrm{~N}$ Cristian. ${ }^{1}$ Pediatric Clinic; ${ }^{2}$ Research, Pediatric Hospital, Lucian Blaga University Sibiu; ${ }^{P}$ Pediatric Hospital, Lucian Blaga University Sibiu; "Pneumology; ${ }^{5}$ Laboratory Research, Pediatric Hospital, Lucian Blaga University Sibiu; 'Research, Pediatric Hospital Sibiu; '7pplied Informatics, Faculty of Science, Lucian Blaga University Sibiu; ${ }^{8}$ Agricultural Sciences and Food Engineering, Faculty of Agricultural Sciences, Food Industry and Environment Protection, Lucian Blaga University Sibiu, Sibiu, Romania

Background Spirometry and biomarkers such as immunoglobulin E (IgE) levels, fractional exhaled nitric oxide concentration (FeNO) have been proposed for assessing the risk of future adverse events in Expert Panel Report 3 (US National Asthma Education and Prevention Program).

Objectives Evaluating the interrelationship between spirometry parameters and/or biomarkers levels (IgE, FeNO, blood eosinophils percentage) and specific symptoms in asthmatic children between hospitalization episodes.

Material and Methods 40 asthmatic pacients were evaluated performing spirometry and measuring biological parameters (Ig E levels, blood eosinophils, Fe NO). Symptoms like cough, dispnoea, wheezing described in patients diaries were analysed in correlation with the spirometric and biological parameters using t-Test, MannWhitney test, Pearson correlations. For each symptom analysed, every time two groups have emerged (with symptoms or without symptoms).

Results Blood eosinophils were different for each symptom, $\mathrm{p}<0.05$ comparing each time the groups. The tendency for positive corelations $\mathrm{p}=0.08$ was noticed between FeNO and FEV1 (forced expiratory vital capacity). Positive corelations, $\mathrm{p}<0.05$ were found between studied parameters, most of the time in the same group (either the group with symptoms or the group without symptoms). 